A left lateral fault with a displacement of 138 nautical miles is required to match the pattern. The goodness of fit shows that the amount of distortion accompanying this left lateral slip over a length of 90 miles was less than 10 per cent and implies a remarkable freedom of movement of one crustal block with respect to the other.

The measured displacements along the Murray Fracture Zone and the Pioneer Ridge reveal an unsuspected mobility of small blocks of oceanic erust.

This work was sponsored by the U.S. Office of Naval Research under Contract No. 2216(05).

University of California

Marine Physical Laboratory,

Scripps Institution of Oceanography,

San Diego 52, California. Dec. 30.

${ }^{1}$ Menard, H. W., Geol. Soc. Amer. Bull., 66, 1188 (1955).

${ }^{2}$ Menard, H. W., and Vacquier, V., "Magnetic Survey of Part of the Deep Sea Floor off the Coast of California", Res. Rev. (Office of Naval Research, June 1958).

${ }^{3}$ Mason, R. G., Geophys. J. (in the press).

\section{Planetary Nebulæ in the Large Magellanic Cloud}

Planetary nebulæ have been found in the Andromeda Nebula ${ }^{1}$ and in the Small Magellanic Cloud ${ }^{2}$. A new catalogue of emission objects in the Small Cloud to be published shortly by me contains nearly six hundred objects; fifty-three of these are planetary nebulæ. Using the Armagh-Dunsink-Harvard telescope and prism with $103 a . E$ red-sensitive plates exposed through a red filter, a planetary nebula is readily recognized by a single $H_{a}$ emission line with no continuum and not associated with nebulosity.

This method has now been applied to the Large Magellanic Cloud. The observational programme is not quite completed and the plates taken have been only provisionally examined. Nearly one thousand emission objects have been found on these plates; at least 120 of these are planetary nebulæ. Where blue-sensitive prism plates are available and the objects are bright enough confirmation as planetaries is provided by the $\mathbf{N}_{1} \mathbf{N}_{2}$ nebular lines of O III. The presence of planetary nebulæe presents additional evidence of Population II objects in the Large Cloud.

Armagh Observatory,

E. M. LINDSAY

Northern Ireland. Dec. 30.

1 Baade, W., Astrophys. J., 60, 151 (1955).

${ }^{2}$ Lindsay, E. M., Mon. Not. Roy. Astro. Soc., 115, 248 (1955): 116 , 649 (1956)

${ }^{3}$ Koelbloed, D., Obs., 76, 191 (1956)

\section{Rotational Structure of the 3914 A. $\mathbf{N}_{2}{ }^{+}$ Band in Sunlit Auroræ}

ON the evenings of September 3 and 4, 1958, striking sunlit auroræ were observed from Saskatoon in the western sky. Although no photographs were taken, there can be no doubt about the nature of these displays because they showed the divided rays with greyish-coloured upper segments as described by Störmer ${ }^{1}$, and because of the peculiarities of the spectrum. During each night of the display a plate was obtained with an $f / 0.8$ Schmidt grating spectrograph having a dispersion of $40 \mathrm{~A} . / \mathrm{mm}$., while a third plate was obtained from the displays of both nights with a large $f / 0.8$ spectrograph having a dispersion of $20 \mathrm{~A} . / \mathrm{mm}$. They were set to record the spectrum in the 3500-4500 A. region and the lines of sight of the instruments were directed towards the upper parts of the rays.

The lower dispersion spectra show unusually high relative intensities of the first negative $\mathrm{N}_{2}{ }^{+}$bands for higher vibrational levels; this effect was first observed by Störmer and explained by Bates ${ }^{2}$ as due to an abnormal distribution of the populations of the vibrational levels of $\mathrm{N}_{2}{ }^{+}$resulting from the fluorescent excitation process dominant in sunlit auroræ. All three plates showed a most unusual intensity distribution in the fine structure of the $0,0 \mathrm{~N}_{2}+$ band at $3914 \mathrm{~A}$. A microphotometer tracing of this band as photographed on the high dispersion plate is shown in Fig. la. For comparison a tracing is shown of the band from a normal aurora in Fig. $1 b$. The band from sunlit auroræ is developed to very high rotational quantum numbers, and its intensity distribution is not quite uniform from line to line. The lines of the $P$ branch have crossed the zero gap and underlie the beginning of the $R$ branch. The rotational temperature derived from the band by plotting $\log \left(I / K^{\prime}\right)$ against $K^{\prime}\left(K^{\prime}+1\right)$ was $2,100^{\circ} \mathrm{K}$. The perturbation at the line $R(38)$ is clearly visible.

There are two possible mechanisms which might explain the high rotational temperature. First, the $\mathrm{N}_{2}{ }^{+}$ions might be in radiative equilibrium with the sunlight; this is an extension of Bates's explanation to cover rotational as well as vibrational degrees of freedom. Second, the $\mathrm{N}_{2}{ }^{+}$ground-state might have a rotational distribution corresponding to thermal equilibrium at some high temperature, which might or might not be the temperature of the atmosphere at the height concerned. In either case, as was pointed out by Swings ${ }^{3}$, the presence of Fraunhofer lines in the exciting sunlight would cause the distribution of line intensities to be non-uniform, just as in the spectra of comets ${ }^{4,5}$. The $\mathrm{N}_{2}{ }^{+}$molecule, being homonuclear, has no rotation or vibration-rotation spectra, so that radiative equilibrium would be established easily if collisions were not important. If the first case is to be treated properly, absorption and emission in the $P$ and $R$ branches of all the bands of the system must be considered at once; for the second case only the absorption is important, but some of the higher bands may still contribute since the vibrational excitation is unusually high.

The exciting intensities were estimated from the Utrecht Atlas of the solar spectrum ${ }^{6}$ as fractions of the continuum; the continuum was considered as having a colour temperature of $7,150^{\circ} \mathrm{K}$. as recommended by Minnaert? The first case was treated approximately by including the $(0,0)$ and $(0,1)$ bands which should be the most important; their shares of the excitation are in the ratio of about $3: 1$. For the second case only the $(0,0)$ band was included. It would be expected that inclusion of more bands would tend to smooth out the distribution of intensities obtained. The general trend of the two sets of results was very similar, but there were considerable differences in detail and the second case gave better agreement with the observations. The temperature used was $2,100^{\circ} \mathrm{K}$., as described above; the agreement of the calculated and observed spectra confirmed it.

We conclude that the $\mathrm{N}_{2}{ }^{+}$ions observed had a rotational temperature in the ground-state of $2,100^{\circ} \mathrm{K}$. There seems to be no way of deciding whether this is also the temperature of the atmosphere at the height observed, except perhaps from the fact that the ions do not seem to be in radiative equilibrium with the sunlight. It is conceivable that the high temperature 